\title{
COMENTARIO EDITORIAL: El mejoramiento de la cantidad y la calidad de vida: una tendencia secular
}

Improvement of life expectancy and quality of life: a secular tendency

Adolfo Rubinstein*

\section{Resumen}

Este artículo constituye la primera entrega de una serie dedicada a la "carga de enfermedad" y a las herramientas para la toma de decisiones en salud. Describe la evolución histórica de algunos indicadores de salud clásicos, su relación con las condiciones socioeconómicas y los desafíos pendientes en materia de salud pública que afronta el mundo y en especial Argentina y que serán desarrollados en forma más extensa en las siguientes entregas de EVIDENCIA.

\section{Abstract}

This article is the first part of a series about "Burden of disease" and health decision making tools. It describes the hystorical evolution of some classic health indicators, their association with socioeconomic characteristics and global public health challenges. Argentina`s particular health status will be discussed in next issues of EVIDENCIA.

Palabras clave: salud pública, desarrollo, tendencias históricas. Key words: public health, development, hystoric tendencies.

Rubisntein A. El mejoramiento de la cantidad y la calidad de vida: una tendencia secular. Evid. actual. práct. ambul; 10(1):2-3, ene-feb.2007.

\section{Un caso paradigmático}

Una niña nacida en Argentina en 1905 podía esperar vivir sólo hasta los 40 años. Desde entonces, su expectativa de vida se ha casi duplicado hasta llegar a los 78 años.

\section{¿Qué significa esto para ella?}

La probabilidad de que muera antes de su primer cumpleaños ha disminuido casi diez veces en los últimos 100 años, de 13\% (130 por mil nacidos vivos) a menos de $1,5 \%$ (14 por mil nacidos vivos) en la actualidad. A partir de la edad adulta, esta probabilidad de morir es mucho menor. En efecto, las muertes maternas o en la edad reproductiva, las muertes por tuberculosis $u$ otras enfermedades transmisibles, a pesar de que siguen siendo causas relevantes para la salud pública en nuestro país, no guardan ni remotamente proporción con lo que acaecía hace un siglo. Por ejemplo, las muertes por enfermedades transmisibles (infecciosas) representaban más de $20 \%$ de todas las muertes en Argentina en 1910 y sólo $4 \%$ en la actualidad. Por el contrario, un siglo después y debido a la disminución de las causas de muerte por causas infecciosas, las relacionadas a enfermedades no transmisibles (ENT) representadas por las enfermedades crónicas y las injurias son por lejos, la principal causa de mortalidad'. En nuestro país en el año 2004, sobre un total de 294.051 muertes se produjeron 90.993 por causas cardiovasculares y 57.772 por cáncer. Ambos grupos de causas constituyen hoy poco más del $50 \%$ de las muertes en Argentina ${ }^{2}$.

Paralelamente, se observan en las últimas décadas marcados mejoramientos en su calidad de vida. Cuando sea adulta, esta niña podrá elegir tener menos niños y por lo tanto pasar menos tiempo embarazada o en período de crianza. De un promedio de más de cinco niños por mujer hace aproximadamente un siglo, hoy esa tasa se ha reducido a menos de la mitad, siendo actualmente de 2,3 .

Esta niña, comparada con su homónima de hace 100 años, tendrá menos enfermedades infecciosas, menos anemia y otros trastornos carenciales, tendrá más fuerza y más estatura, una mente más rápida y muchísimas más posibilidades de alcanzar un desarrollo pleno en su vida familiar y laboral. Su vida no solo será más larga sino también mucho más saludable.

Esta historia que refleja los enormes avances en el mejoramiento de la salud en nuestro país no es diferente a lo que ha ocurrido en la mayoría de los países del mundo. Es más, muchas de estas mejorías han sido posibles sin un dramático incremento de los ingresos económicos promedio de la población. Por ejemplo, los niveles de ingresos en los Estados Unidos en las primeras décadas del siglo XX no eran muy diferentes a los que hoy tiene Argentina, aún cuando la expectativa de vida en Estados Unidos en esa época era 25 años más corta.

\section{Mejoramientos de la salud}

El siglo 20 será recordado como el período en el que ha ocurrido el mayor incremento de la expectativa de vida en la historia humana. Si buceamos un poco más en un pasado no tan lejano, podemos observar que en los últimos 150 años, la expectativa de vida en los países más ricos y saludables se ha incrementado sostenidamente. Desde el comienzo de los registros de mortalidad en Inglaterra y Gales hacia 1640 y hasta 1840 se aprecian algunas fluctuaciones sin una clara tendencia, pero después de 1840 la curva de expectativa de vida sube en los países ricos aceleradamente a un ritmo constante de casi 2,5 años por década hasta el presente. Hay clara evidencia empírica de que la reducción dramática de la mortalidad en los países desarrollados ocurridos en los siglos XIX y la primera mitad del siglo XX se relacionaron fundamentalmente con el mejoramiento del ingreso económico, las condiciones de vida y las condiciones sanitarias, la nutrición y la educación, antecediendo a los avances científicos de la medicina y la salud pública ${ }^{3}$. Sin embargo hay también evidencia de que en los países de bajos y medianos ingresos la reducción de la mortalidad en las últimos cuatro décadas se debió en un $50 \%$ al progreso científico y tecnológico, en un $40 \%$ a la educación de las mujeres en edad reproductiva y en un $20 \%$ al mejoramiento del ingreso (World Development Report 1999).

Además, a partir de la segunda mitad del siglo 20 , las diferencias en la expectativa de vida entre estos países y los países más pobres se han reducido notablemente. A pesar de esto, la expectativa promedio al nacer del percentilo $50 \%$ de los países en el mundo se aproxima hoy a la expectativa de vida que los países nórdicos tenían en las primeras décadas del siglo pasado. Sin embargo, desde 1950 este indicador en los países de ingresos medios viene convergiendo lentamente a los máximos actuales, contrastando paradójicamente con el aumento en las desigualdades económicas entre los países y al interior de los mismos.

Por lo tanto, las razones por las que persisten estas desigualdades sanitarias descansan sólo parcialmente en las desigualdades en el ingreso económico: las experiencias de China, Costa Rica, Cuba, Sri Lanka y el estado de Kerala en la India, entre otros, muestran de manera concluyente que el mejoramiento de las condiciones sanitarias no está necesariamente relacionado con igual mejoramiento de las condiciones económicas. Sólo en Latinoamérica, la expectativa de vida promedio ha aumentado desde 56 años en 1960 a 71 años en 2002 con un crecimiento del $4 \%$ anual entre 1960 y 1990 y del $2,5 \%$ en la década siguiente hasta la actualidad. Este fenómeno puede ser explicado fundamentalmente por la expansión y difusión del conocimiento y la tecnología -uno de los

Médico Especialista en Medicina Familiar, Profesor y Doctor en Medicina. Jefe del Servicio de Medicina Familiar del Hospital Italiano de Buenos Aires. 
grandes logros de la globalización- y por las intervenciones directas en salud pública. A pesar de estos importantes logros, el 50\% de todas las muertes en el mundo, que además afectan preponderantemente a los niños, aún son debidas a diarrea e infecciones respiratorias, siete millones de adultos mueren de condiciones que podrían ser curadas sin mucho costo (dos millones por tuberculosis) y medio millón de mujeres mueren por complicaciones directas del parto. Para peor, la tasa de mortalidad infantil es diez veces mayor en los países subdesarrollados que en los desarrollados y la tasa de mortalidad materna, 30 veces mayor. En definitiva, si todos los países del mundo tuvieran la tasa de mortalidad de los menores de cinco años de Japón (la más baja del mundo) se evitarían 11 millones de muertes infantiles cada año. La mortalidad materna es otro factor preocupante a pesar de que sólo constituya alrededor de $1 \%$ de todas las muertes globales ya que su incidencia oscila entre $6 / 100.000$ en Australia a 1800/100.000 en Sierra Leona, siendo en nuestro país de 44/100.000 para 2003.

Además, la expectativa de vida al nacer puede disfrazar pequeñas diferencias en la duración de la vida a edades mas avanzadas. Para la gente que alcanza la vida adulta en los países en desarro-
Ilo, su expectativa de vida comienza a aproximarse a las de los individuos que viven en países desarrollados. Sin embargo, para la misma edad, la probabilidad de envejecimiento funcional o discapacidad es mucho mayor en estos últimos países.

\section{Los desafíos pendientes}

Como hemos visto, si bien han podido observarse progresos importantes en los últimos tiempos, existen todavía desafíos globales que todos los países y en especial los de nuestra región deberán enfrentar, para lo cual es necesario implementar políticas de salud apropiadas. Dentro de los principales desafíos que los países periféricos deben abordar, se encuentran el modo de hacer frente a la transición epidemiológica y la epidemia de enfermedades crónicas, pero además lograr que los avances científicos y tecnológicos no sólo mejoren los indicadores sanitarios en promedio, sino que lleguen a toda la población con adecuados niveles de calidad y cobertura, procurando el mínimo de inequidad posible.

Recibido el 20/12/06 y aceptado el 27/02/07.

\section{Referencias}

1. Pantelides EA. La Transición Demográfica Argentina: Un Modelo no Ortodoxo. Desarrollo Económico. 1983; $12: 1-25$.

2. Ministerio de Salud de la Nación y OPS/OMS. Indicadores Básicos Argentina 2005. Publicación del Ministerio de Salud de la Nación y OPS/OMS.

3. McKeown T, Record RG. Reasons for the decline in mortality in England and Wales during the nineteenth century. Population Studies, 1962, 16: 94-122.

\section{INFO - EVIDENCIA}

\section{¿Qué es Evidencia, Actualización en la Práctica Ambulatoria?}

Una publicación independiente editada cada dos meses desde 1997 por la Fundación MF, Organización sin fines de lucro dedicada a promover el desarrollo de la medicina familiar y la atención primaria de la salud.

\section{¿Cuál es el objetivo de Evidencia?}

Contribuir a la educación continua y a la actualización de los profesionales de la salud de la región en el área de la atención ambulatoria.

\section{¿Contenidos de Evidencia}

- Información independiente, actualizada y resumida en forma sencilla y en castellano sobre temas relevantes de la atención ambulatoria.

- Artículos seleccionados por su calidad y relevancia clínica, resumidos y comentados críticamente por destacados profesionales del área.

- Revisiones sobre temas clínicos, epidemiológicos, sanitarios o humanísticos, de importancia para la práctica ambulatoria.

- Notas farmacológicas para la actualización rápida de temas de terapéutica clínica.

- Discusión de casos clínicos con herramientas de Medicina Basada en la Evidencia

- Glosario de términos de epidemiología y medicina basada en la evidencia

¿Cómo es posible acceder a Evidencia?

Por suscripción a la edición en papel, o bien por Intenet, en nuestro sitio:

http://www.evidencia.org 